\title{
RAP-PCR fingerprinting reveals time-dependent expression of matrix-related molecules following stem-cell based TGF $\beta 1$-induced chondrocyte development
}

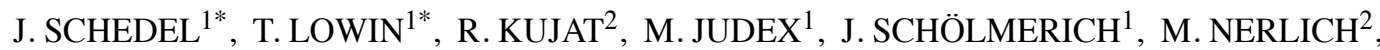 \\ U. MÜLLER-LADNER ${ }^{1,3}$ and P. ANGELE ${ }^{2}$
}

\begin{abstract}
Departments of ${ }^{1}$ Internal Medicine I and ${ }^{2}$ Trauma Surgery, University Hospital of Regensburg; ${ }^{3}$ Department of
\end{abstract} Rheumatology and Clinical Immunology, Kerckhoff Clinic Bad Nauheim, University of Giessen, Germany

Received November 2, 2010; Accepted December 27, 2010

DOI: $10.3892 /$ ijmm.2011.608

\begin{abstract}
Different approaches of engineering cartilage to treat defects in the articulating surfaces of the joints have been designed, which mainly use mesenchymal stem cells or autologous chondrocytes for in situ transplantation. However, these cells are poorly characterized with respect to viability, degree of differentiation and morphology or production of extracellular matrix. At present, one of the key approaches to generate chondrocytes is the stimulation of stem cells with transforming growth factor (TGF) $\beta 1$. To characterize the molecular alterations occuring during the cellular transformation induced by TGF- $\beta 1$ exposure, the differentiation process of bone marrow-derived stem cells into chondrocytes was investigated using an in vitro chondrogenesis model and the RNA arbitrarily primed PCR (RAP-PCR) fingerprinting technique. Distinct genes were found to be differentially regulated during chondrocyte development beginning on day 1: collagen type I, non-muscle myosin MYH9, followed by manganese superoxide dismutase and sodium-potassium ATPase on day 7. The results suggest that using RAP-PCR for differential display fingerprinting is a useful tool to investigate the differentiation process of bone marrow-derived stem cells following TGF- $\beta 1$-stimulation.
\end{abstract}

\section{Introduction}

Cartilage lesions are a consequence of etiologically and pathogenetically different primary and secondary disease entities of the joint. The pathophysiological problem of these

Correspondence to: Dr Joerg Schedel, Present address: ${ }^{4}$ Department of Internal Medicine I, Hospital of Weiden, Soellnerstrasse 16, D-92637 Weiden, Germany

E-mail: joerg.schedel@googlemail.com

*Contributed equally

Key words: RNA arbitrarily primed PCR, TGF $\beta 1$, bone marrowderived stem cells, chondrocytes lesions is based on the insufficient regenerative capabilities of cartilage to synthesize and integrate the required macromolecular components in the appropriate architecture (1).

Different surgical procedures have been implemented for the treatment of cartilage defects. These methods are mainly directed toward accessing the subchondral vasculature and bone marrow either by abrasion of the cartilage and subchondral bone plate (2), by drilling of the subchondral bone (3) or by microfracturing (4). However, the fibrocartilage-like reparative tissue that results from these techniques lacks the composition, structure, and mechanical properties of the normal cartilage, and the long-term clinical outcomes are unpredictable (5). Patients treated with these techniques frequently experience short-term pain relief but develop progressive osteoarthritic symptoms when the reparative tissue disintegrates (4). In addition, various surgical procedures have been developed to deliver autologous chondrogenic cells to cartilage using cell suspensions prepared by the expansion of cells obtained from a cartilage biopsy (6), from precursor cells derived from the periosteum (7) or from the perichondrium (8) with the latter eventually undergoing terminal differentiation into chondrocytes in situ.

In recent years, variable approaches to engineer cartilage (tissue engineering) have been undertaken using mesenchymal stem cells (MSC) (9) or autologous chondrocyte transplantation (10). Yet the problems of a sufficient attachment of these cells in the cartilage lesion, the maintenance of their biomechanical properties for an extended period of time, and the molecular basis for their potential rejection remain unresolved. These issues are based on the fact that the cells are insufficiently characterized with respect to their viability, degree of differentiation, morphology or production of extracellular matrix, although, for example, bone marrowderived mesenchymal progenitor cells have been ascribed osteochondral differentiation potential in vitro (11) and in vivo (12), and may therefore, play an important role in the repair of extracellular matrix defects.

Among the current tissue engineering strategies to provide cartilaginous or chondrocyte-like cells for potential repair of articular defects, transforming growth factor (TGF)- $\beta 1$ induced differentiation of stem cells into chondrocyte-like 
cells is one of the most promising approaches (13). An in vitro chondrogenesis model established in our laboratory provides a useful model for studying cellular differentiation processes (14). As little is known about the time-dependent molecular alterations inherent with the cellular transformation stimulated by the TGF- $\beta 1$ gene transfer, we evaluated which genes are up-regulated in bone marrow-derived stem cells during their maturation into chondrocytes.

\section{Materials and methods}

Cell culture. Human bone marrow was obtained from the iliac crest of a patient undergoing spine fusion using a protocol approved by the Ethics Committee and the Institutional Review Board of the University Hospital of Regensburg, Germany. After Percoll (GE Healthcare Europe, Freiburg, Germany) gradient fractionation, Dulbecco's Modified Eagle's Medium with $10 \%$ fetal bovine serum (PAN, Aidenbach, Germany) was added to the aspirate and $10 \times 10^{6}$ nucleated cells $/ 100 \mathrm{~mm}^{2}$ dish were plated and grown at $37^{\circ} \mathrm{C}$ with $5 \% \mathrm{CO}_{2}$ until $80 \%$ confluency.

In vitro chondrogenesis. Adherent cell colonies were trypsinized, counted, and $2 \times 10^{5}$ cell aliquots were spun down in $15 \mathrm{ml}$ polypropylene conical tubes in a defined medium as previously demonstrated to induce the chondrogenic differentiation of the bone marrow cells in this culture system $(14,15)$. Aggregate cultures were obtained by addition of TGF- $\beta 1$ (10 ng/ml, R\&D Systems, Wiesbaden, Germany). Control replicates were cultured without TGF- $\beta 1$. Culture medium was changed every 3 days. Samples were harvested at days 0 , $1,7,14$ and 21 of culture.

Immunohistology and immunohistochemistry. Aggregates were fixed and embedded in paraffin. Serial sections $(5 \mu \mathrm{m})$ of all samples were cut and stained with toluidine blue for histological evaluation. For immunohistochemical analysis, unstained sections were deparaffinized. After blocking of non-specific antibody binding with isotype matched $\mathrm{IgG}$, the sections were digested for 30 min with $1 \%$ pronase and $0.1 \mathrm{U} / \mathrm{ml}$ chondroitinase ABC (Seikagaku, Tokyo, Japan) to facilitate the access of anti-collagen antibodies to immunoreactive epitopes. Thereafter, the sections were probed with anti-collagen antibodies raised against human type II collagen (II-II6B3, Developmental Studies Hybridoma Bank, University of Iowa and Johns Hopkins University Baltimore, USA). Visualization was achieved using FITC-conjugated secondary antibodies (goat anti-mouse IgG, Cappel, Aurora, $\mathrm{OH}, \mathrm{USA}$ ), and the samples were investigated by two different examiners using a Leitz photomicroscope (Leitz, Wetzlar, Germany).

Aggregates were fixed in formaldehyde, embedded in Tissue-Tek O.C.T. compound (Sakura Finetek, Zoeterwoude, The Netherlands) and frozen in liquid nitrogen. Cryosections $(10 \mu \mathrm{m})$ were stained with $0.1 \%$ aqueous toluidine blue for histological evaluation. Expression of collagen type II was evaluated by the immunoperoxidase ABC technique (Vector, Burlingame, CA, USA) according to Hsu et al (15), after predigestion of sections (15 min in $0.1 \%$ pepsin at $\mathrm{pH} 3.5$ ), and incubation with monoclonal primary mouse anti-human collagen type II antibodies (clone II-4C11; Calbiochem-Merck, Schwalbach, Germany), followed by biotin-conjugated polyclonal secondary goat anti-mouse IgG antibodies (Jackson, West Grove, PA, USA), and the Ni and Co enhanced DAB stain visualization. Immunostained sections were counterstained with azocarmine.

RNA extraction. Total cellular RNA of cultured bone marrow cells was extracted on days 1, 7, 14 and 21 using the RNeasy spin column purification kit (Qiagen, Hilden, Germany) in combination with the RNase-free DNase Set (Qiagen). RNA concentrations were measured using the Ribogreen RNA quantification kit (Molecular Probes, Leiden, The Netherlands), and stored at $-70^{\circ} \mathrm{C}$.

RNA arbitrarily primed PCR (RAP-PCR) of total cellular $R N A$. RAP-PCR of total cellular RNA was performed as previously described $(16,17)$. First strand synthesis was carried out using MuLV reverse transcriptase (Promega, Madison, WI, USA), $250 \mathrm{ng}$ of RNA and $2 \mu \mathrm{M}$ first strand arbitrary 10-base primers (OPN23: 5'-CAG GGG CAC C-3' and OPN29: 5'-CAC CAG GGG C-3'). Second strand synthesis was performed for 35 low stringency cycles $\left(30 \mathrm{sec} 94^{\circ} \mathrm{C}, 30 \mathrm{sec} 35^{\circ} \mathrm{C}, 30 \mathrm{sec}\right.$ $72^{\circ} \mathrm{C}$ ) using the AmpliTaq Stoffel Fragment (Perkin-Elmer, Norwalk, CT, USA) and $4 \mu \mathrm{M}$ of two different second arbitrary 10-base primers (OPN21: 5'-ACC AGG GGC A-3' and $\mathrm{KinA1}^{+}$: 5'-GAG GGT GCC TT-3'). PCR reactions (2 $\left.\mu \mathrm{l}\right)$ were separated on an $8 \mathrm{M}$ urea/6\% polyacrylamide gel and electrophoresed. Electrophoresis was performed for $4-6 \mathrm{~h}$ at $50 \mathrm{~W}$ in $1 \mathrm{X}$ Tris-borate EDTA buffer. Gels were then transferred to $3 \mathrm{MM}$ Whatman paper, dried under vacuum at $80^{\circ} \mathrm{C}$, and directly exposed to Kodak BioMax ${ }^{\mathrm{TM}}$ autoradiography film (Kodak, Stuttgart, Germany) at room temperature for 12-72 h, depending on the intensity of radiation of the amplified fragments. Several luminescence labels (autoradiogram markers; Stratagene, San Diego, CA, USA) were attached to the gel to facilitate alignment of the autoradiograms with the gels for subsequent isolation of differentially displayed gene fragments.

Isolation and purification of differentially amplified polymerase chain reaction products. Gel slices carrying the target fragment were then excised with a razor blade and placed in $50 \mu \mathrm{l}$ TE buffer $(10 \mathrm{mmol} / \mathrm{l}$ Tris- $\mathrm{HCl}, 1 \mathrm{mmol} / \mathrm{l}$ EDTA, $\mathrm{pH}$ 8.0). DNA was eluted by incubation at $65^{\circ} \mathrm{C}$ for $3 \mathrm{~h}$. Eluates were taken for re-amplification of the gene fragment using the primers of the original fingerprint and the conditions outlined above for 20 cycles. PCR products were routinely checked by denaturing polyacrylamide gel electrophoresis, loading the re-amplified product next to the original fingerprint to verify its size and purity. To exclude contamination of nondifferentially regulated gene products of similar size to that of the regulated transcripts, native polyacrylamide gels were used to separate the sequences of the re-amplified mixture based on single-strand confirmation polymorphism (SSCP) as previously described (18). After a second identification of the gene segment using this procedure, it was cut from the SSCP gel and re-amplified a second time.

Cloning, Southern blot analysis and sequencing. After verifying its correct size and purity on $4 \%$ agarose gels, the 
re-amplified products derived from the SSCP gel were cloned into PCR ${ }^{\circledR}$-II Topo using the TOPO-TA-Cloning ${ }^{\circledR}$ Kit Dual Promoter (Invitrogen, De Schelp, The Netherlands). After blue-white screening of the clones, 10 white colonies and one blue colony were picked and suspended in $50 \mu \mathrm{l}$ water. Aliquots of the bacterial suspensions were checked by high-stringency PCR for the presence and the correct length of inserts using the $\mathrm{T} 7$ and the M13 (or M20) reverse sequencing primers. Clones of the correct length were subsequently grown overnight in $5 \mathrm{ml} \mathrm{LB}$ medium containing $50 \mu \mathrm{g} / \mathrm{ml}$ of ampicillin for plasmid isolation. Five clones per transcript were sequenced with the Applied Biosystems 373 automatic sequencer using the Perkin-Elmer (Norwalk, CT, USA) DNA sequencing kit. The databases of the National Center for Biological Information were screened to align the obtained sequences with known complementary DNA clones, genomic clones and cloned expressed sequence tags. If sequences were represented multiple times and confirmed within the majority of the clones resulting from one RAP-PCR product, inserts were re-amplified and used as probes against Southern blots of the original fingerprint gel. This procedure confirms that the selected sequences were in fact differentially amplified in the original fingerprint gels. For this, DNA from RAP-PCR fingerprint gels was transferred to nylon membranes (Duralon-UV, Stratagene, San Diego, CA, USA) by capillary action in a 10X saline sodium citrate (SSC) buffer overnight. After ultraviolet cross-linking, blots were pre-hybridized, hybridized and analyzed using established protocols (19).

\section{Results}

Chondrogenic differentiation of bone marrow cells using $T G F-\beta 1$. Human bone marrow-derived aggregate cultures were incubated with and without TGF- $\beta 1$. Without addition of TGF- $\beta 1$, no chondrogenesis could be detected in the aggregates using toluidine blue staining. With addition of TGF- $\beta 1$, chondrocytic differentiation was first observed on day 14 and collagen type II was synthesized in substantial amounts in the aggregates within 21 days (Fig. 1).

Molecular analysis of TGF- $\beta 1$-treated or untreated bone marrow cells using RAP-PCR and cDNA arrays. After RNA isolation and RAP-PCR fingerprint analysis, approximately 50 different RNAs were obtained per primer pair at each time point of the analysis (i.e., days 1, 7, 14 and 21), of which most were expressed in bone marrow-derived cells after TGF- $\beta 1$ stimulation. Re-amplification of differentially expressed RAP-PCR products and gene sequence analysis revealed distinct genes potentially associated with chondrogenesis that were specifically up-regulated following TGF- $\beta 1$ addition. In total, 24 clones were analyzed, and 4 genes were found to be up-regulated. On day 1, collagen type I, a marker of initiation of matrix production, and non-muscle myosin MYH9, an indicator for cellular proliferation and differentiation of the cytoskeleton were found to be up-regulated. On day 7, manganese superoxide dismutase (MnSOD) and sodium-potassium ATPase, a marker for enhanced cellular metabolism and intracellular enzyme activity were shown to be up-regulated (Fig. 2). After day 14, using the same primer sets for RAP-PCR as those for days 1 and 7, none of the amplified segments were

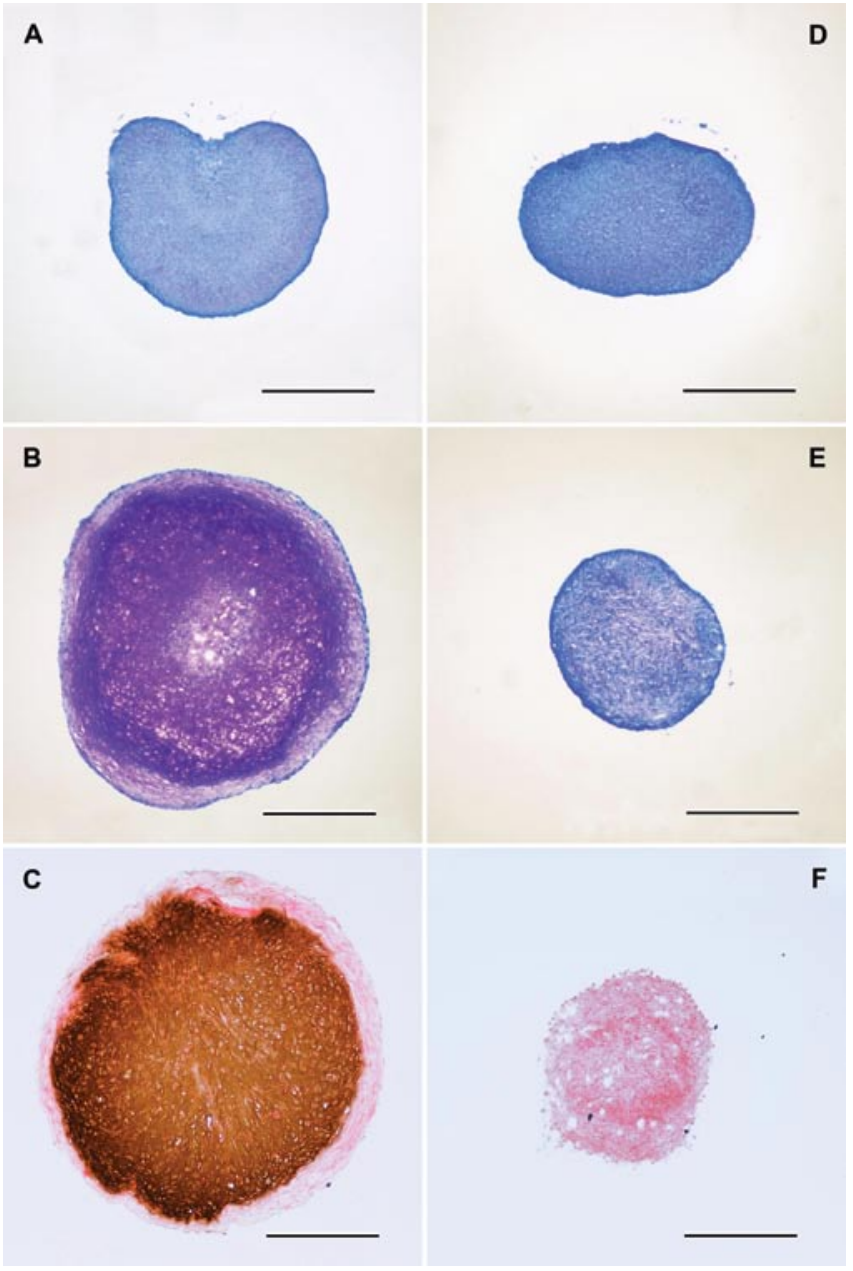

Figure 1. Histology (toluidine blue; A, B, D, E) and immunohistochemistry (collagen type II; C, F) of aggregates after 1 day (A, D) and 21 days $(\mathrm{B}, \mathrm{C}$, $\mathrm{E}, \mathrm{F})$ of culture. Addition of TGF- $\beta 1(\mathrm{~A}, \mathrm{~B}, \mathrm{C})$ resulted in chondrogenic differentiation, indicated by metachromatic staining of the extracellular matrix with toluidine blue (B) and by a strong collagen type II immunoreactivity (C) after 21 days. Aggregates cultured without TGF- $\beta 1$ addition show no chondrogenesis (D, E, F). Bars represent $300 \mu \mathrm{m}$.

specific for TGF- $\beta 1$-treated cells indicating a substantially different gene expression in the terminal phases.

\section{Discussion}

Among the tissue engineering strategies to potentially repair articular defects, differentiation of bone marrow-derived stem cells into chondrocyte-like cells is one of the most promising approaches (20). Several growth factors are known to be able to drive this differentiation process, such as transforming growth factors (TGF- $\beta 1,-2$, and -3) $(21,22)$. Using TGF- $\beta 1$, the detection of collagen types II and X mRNA has been described and represents the strongest hint that the formed matrix is at least cartilage-like (14). This finding is underlined by the results of the present study showing that without addition of TGF- $\beta 1$, chondrogenesis, as characterized by collagen type II expression, can not be observed.

However, in some studies investigating the implantation of these progenitor cells into cartilage defects, a hyaline-like cartilage could not be generated (23). Consequently, the question arose which factors may be lacking in the healing process 


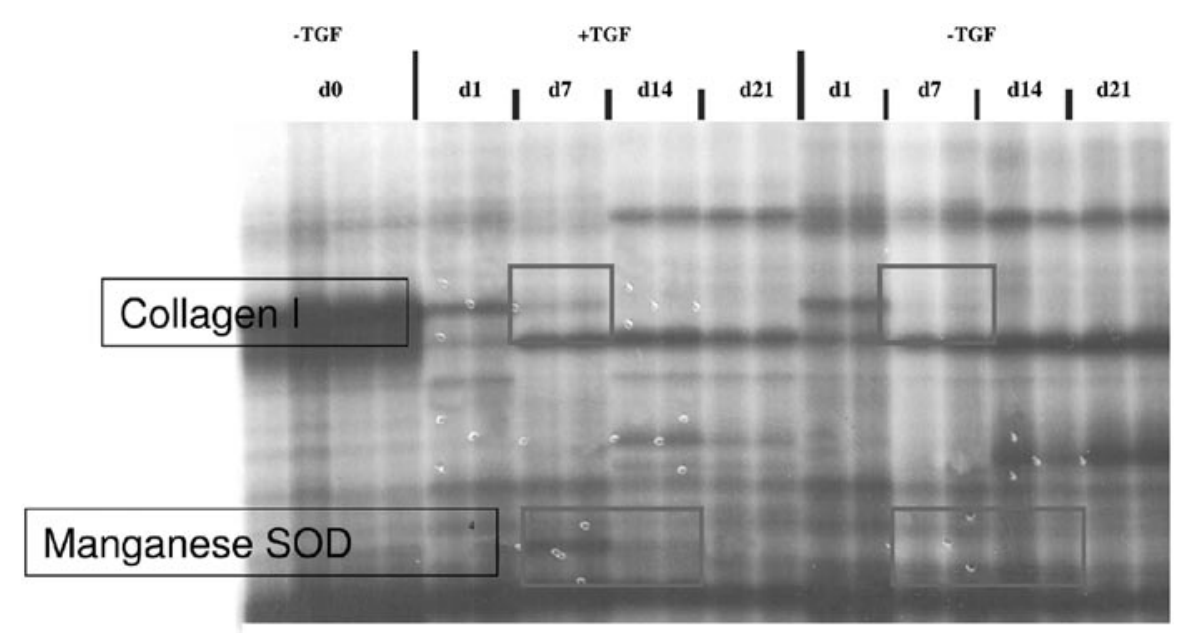

Figure 2. Representative example of a RAP-PCR fingerprint after OPN23/OPN29 amplification of the RNA of chondrocytic aggregates \#LN40 during chondrogenesis. Of the potentially up-regulated identified gene sequences in this experiment, 3 of the 4 genes could be re-identified after confirmation by gene sequence analysis. 1, collagen type I, with a decrease on day 7; 2, manganese superoxide dismutase (SOD); and 3, sodium-potassium ATPase (not shown). Differentially regulated genes are marked by frames.

that could permit the differentiation into a cartilage-like matrix, and to be able to visualize the gene expression during this process. For this purpose, an in vitro chondrogenesis model was established in our laboratory, which provides a useful model for studying the cellular differentiation processes (14). Different techniques are available to compare differences in gene expression, and for most of them, large amounts of mRNA are necessary in order to detect distinct differences in expression (e.g., subtractive hybridization). When evaluating slowly proliferating cells such as synovial fibroblasts or chondrocytes, however, only limited amounts of RNA are available. For these cells, RAP-PCR has proven to be both efficient and reliable for examining differentially regulated genes $(16,24,25)$.

Using this technique, we have identified a differential gene expression pattern by demonstrating that collagen type I and non-muscle myosin MYH9 on day 1, and MnSOD and sodium-potassium ATPase on day 7, were differentially up-regulated following TGF- $\beta 1$ stimulation. Whereas collagen type II is the most prominent and important component of human healthy articular cartilage, collagen type I is produced in osteoarthritic cartilage by chondrocytes attempting to compensate for the cartilage matrix loss. Our results seem to be in contrast to recent findings showing a decreased expession of collagen type I and an increase in collagen type II in bone marrow-derived MSC differentiated in pellet mass cultures, but most probably reflect the different cell population investigated (MSC vs. bone marrow cells in toto) (26).

Non-muscle myosin MYH9 is a member of the myosin super-family of proteins which share functional properties involved in cellular movement, cell division or muscle contraction. Whereas mutations in the MYH9 gene have commonly been associated with a clinical syndrom comprising nephropathy and macrothrombocytopenia (27), recently Takubo et al reported that MYH9 colocalizes with actin stress fibers indicating a function in the cytosekeletal structure in mammalian cells (28). Since the intracellular structure is of considerable importance for both intra- and extracellular functions of the cells, this differentially-regulated gene appears to represent early changes in the differentiation process of the cell.

MnSOD is one of several dismutases exerting an antioxidant function with respect to reactive oxygen species (ROS) induced by pro-inflammatory cytokines, and participates in the matrix-degrading activity of both inflammatory and non-inflammatory joint diseases. We found elevated MnSOD mRNA expression in chondrocytes after TGF- $\beta 1$ induction. This findings is in line with results from Mazzetti et al who reported up-regulation of intracellular MnSOD in osteoarthritic chondrocytes in response to several cytokines; this up-regulation was interpreted as a compensatory reaction to the higher nitric oxide levels in osteoarthritis chondrocytes compared to rheumatoid arthritis chondrocytes (29). Moreover, superoxide dismutase has been shown to effectively prevent interleukin- $1 \beta$ IкB $\alpha$ degradation and, thus, inhibit inducible nitric oxide synthase (iNOS) expression (30), as well as production of ROS induced by tensile stress (31). Taken together, inceased MnSOD expression could result in a higher capacity of chondrocytes to counter-balance elevated ROS levels immanent in the pathological condition of altered articular cartilage.

To balance the negative charges of proteoglycans in the extracellular matrix, sodium-potassium-ATPases in the plasma membrane of chondrocytes are required. They are able to regulate the intra- and extracellular exchange of $\mathrm{Na}$ and $\mathrm{K}$, and several isoforms of this enzyme reflecting differential cationic environmental changes and enzymatic activity under arthritic conditions have been described $(32,33)$. In line with these regulatory functions, up-regulation of the enzyme sodium-potassium-ATPase could reflect the arthritic condition as such, or the requirement to counter-balance the ionic or osmotic imbalance within the extracellular articular matrix as seen in osteoarthritis or other conditions in which the articular cartilage has been damaged.

In conclusion, the data suggest that distinct genes involved in the cellular metabolism and matrix-production may specifically be up-regulated at different time points in chondrocyte differentiation induced by TGF- $\beta 1$. Moreover, the results 
confirm that using RAP-PCR for differential display fingerprinting is an efficient and sensitive tool to investigate the differentiation process of bone marrow-derived stem cells, for instance after TGF- $\beta 1$ stimulation. Further analysis of the expression patterns detected by this technique could provide additional data to characterize the chondrocytic cell type needed for long-term production of an articular cartilage-like matrix.

\section{Acknowledgements}

The authors would like to thank Wibke Ballhorn for excellent technical assistance. This study was supported by the German Scientific Research Community (DFG grants Sche 671/5-1, Mu1383/3-3 and Mu 1383/9-1).

\section{References}

1. Ghivizzani SC, Oligino TJ, Robbins PD and Evans CH: Cartilage injury and repair. Phys Med Rehabil Clin N Am 11: 289-307, 2000.

2. Matsunaga D, Akizuki S, Takizawa T, Yamazaki I and Kuraishi J: Repair of articular cartilage and clinical outcome after osteotomy with microfracture or abrasion arthroplasty for medial gonarthrosis. Knee 14: 465-471, 2007.

3. Müller B and Kohn D: Indication for and performance of articular cartilage drilling using the Pridie method. Orthopäde 28: 4-10, 1999 (In German).

4. Spahn G, Mueckley T, Kahl E and Hofmann GO: Factors affecting the outcome of arthroscopy in medial-compartment osteoarthritis of the knee. Arthroscopy 22: 1233-1240, 2006.

5. Johnson LL: Arthroscopic abrasion arthroplasty: a review. Clin Orthop Relat Res 391 (Suppl): S306-S317, 2001.

6. Tallheden T, Brittberg M, Peterson L and Lindahl A: Human articular chondrocytes-plasticity and differentiation potential Cells Tissues Organs 184: 55-67, 2006.

7. O'Driscoll SW: Articular cartilage regeneration using periosteum. Clin Orthop Relat Res 367 (Suppl): S186-203, 1999.

8. Bruns J and Steinhagen J: Treatment of deep hyalin cartilage defects with autologous perichondrial grafts. Int J Sports Med 24: 382-388, 2003.

9. Mobasheri A, Csaki C, Clutterbuck AL, Rahmanzadeh M and Shakibaei M: Mesenchymal stem cells in connective tissue engineering and regenerative medicine: applications in cartilage repair and osteoarthritis therapy. Histol Histopathol 24: 347-366, 2009.

10. Brittberg M: Autologous chondrocyte implantation-technique and long-term follow-up. Injury 39 (Suppl. 1): S40-S49, 2008.

11. Bernardo ME, Emons JA, Karperien M, Nauta AJ, Willemze R, Roelofs H, Romeo S, Marchini A, Rappold GA, Vukicevic S, Locatelli F and Fibbe WE: Human mesenchymal stem cells derived from bone marrow display a better chondrogenic differentiation compared with other sources. Connect Tissue Res 48: 132-140, 2007.

12. Yoo JU, Barthel TS, Nishimura K, Solchaga L, Caplan AI, Goldberg VM and Johnstone B: The chondrogenic potential of human bone-marrow-derived mesenchymal progenitor cells. J Bone Joint Surg 80: 1745-1757, 1998.

13. Darling EM and Athanasiou KA: Biomechanical strategies for articular cartilage regeneration. Ann Biomed Eng 31: 1114-1124, 2003.

14. Angele P, Yoo JU, Smith C, Mansour J, Jepsen KJ, Nerlich M and Johnstone B: Cyclic hydrostatic pressure enhances the chondrogenic phenotype of human mesenchymal progenitor cells differentiated in vitro. J Orthop Res 21: 451-457, 2003.

15. Hsu SM, Raine L and Fanger H: Use of avidin-biotin-peroxidase complex $(\mathrm{ABC})$ in immunoperoxidase techniques: a comparison between $\mathrm{ABC}$ and unlabeled antibody (PAP) procedures. J Histochem Cytochem 29: 577-580, 1981.
16. Neumann E, Kullmann F, Judex M, Juesten HP, Wessinghage D, Gay S, Schölmerich J and Mueller-Ladner U: Identification of differentially expressed genes in rheumatoid arthritis by a combination of complementary DNA array and RNA arbitrarily primed-polymerase chain reaction. Arthritis Rheum 46: 52-63, 2002.

17. Neumann E, Lechner S, Tarner IH, et al: Evaluation of differentially expressed genes by a combination of cDNA array and RAP-PCR using the AtlasImage 2.0 software. J Autoimmun 21: 161-166, 2003.

18. Mathieu-Daudé F, Cheng R, Welsh J and McClelland M: Screening of differentially amplified cDNA products from RNA arbitrarily primed PCR fingerprints using single strand conformation polymorphism (SSCP) gels. Nucleic Acids Res 24: 1504-1507, 1996.

19. Maniatis T: Molecular Cloning. Protocols and Applications. Raven Press, New York, 1997.

20. Vinatier C, Mrugala D, Jorgensen C, Guicheux J and Noël D: Cartilage engineering: a crucial combination of cells, biomaterials and biofactors. Trends Biotechnol 27: 307-314, 2009.

21. Kawakami Y, Rodriguez-León J and Belmonte JC: The role of TGFbetas and Sox9 during limb chondrogenesis. Curr Opin Cell Biol 18: 723-729, 2006.

22. Ng F, Boucher S, Koh S, et al: PDGF, TGF- $\beta$, and FGF signaling is important for differentiation and growth of mesenchymal stem cells (MSCs): transcriptional profiling can identify markers and signaling pathways important in differentiation of MSCs into adipogenic, chondrogenic, and osteogenic lineages. Blood 112: 295-307, 2008.

23. Goldberg VM, Solchaga L, Lundberg M, et al: Mesenchymal stem cell repair of osteochondral defects of articular cartilage. Semin Arthroplasty 10: 30-36, 1999.

24. Tsunoda T, Nakamura T, Ishimoto K, Yamaue H, Tanimura H, Saijo $\mathrm{N}$ et al: Upregulated expression of angiogenesis genes and down regulation of cell cycle genes in human colorectal cancer tissue determined by cDNA macroarray. Anticancer Res 21: 137-143, 2001.

25. Meyringer R, Neumann E, Judex M, et al: Analysis of gene expression patterns in systemic sclerosis fibroblasts using RNA arbitrarily primed-polymerase chain reaction for differential display. J Rheumatol 34: 747-753, 2007.

26. Karlsson C, Brantsing C, Svensson T, Brisby H, Asp J, Tallheden T and Lindahl A: Differentiation of human mesenchymal stem cells and articular chondrocytes: analysis of chondrogenic potential and expression pattern of differentiationrelated transcription factors. J Orthop Res 25: 152-163, 2007.

27. Basile C, Schiavone P, Heidet L and Grundfeld JP: Hereditary nephritis with macrothrombocytopenia: phenotypic variety and the genotypic defect. J Nephrol 15: 320-323, 2002.

28. Takubo T, Wakui S, Daigo K, Kurokata K, Ohashi T, Katayama K and Hino M: Expression of non-muscle type myosin heavy polypeptide 9 (MYH9) in mammalian cells. Eur J Histochem 47: 345-352, 2003.

29. Mazzetti I, Grigolo B, Pulsatelli L, Dolzani P, Silvestri T, Roseti L, Meliconi R and Facchini A: Differential roles of nitric oxide and oxygen radicals in chondrocytes affected by osteoarthritis and rheumatoid arthritis. Clin Sci (London) 101: 595-599, 2001.

30. Mendes AF, Caramona MM, Carvalho AP and Lopes MC: Differential roles of hydrogen peroxide and superoxide in mediating IL-1-induced NF-kappa B activation and iNOS expression in bovine articular chondrocytes. J Cell Biochem 88: 783-793, 2003

31. Yamazaki K, Fukuda K, Matsukawa M, Hara F, Matsushita T, Yamamoto N, Yoshida K, Munakata $\mathrm{H}$ and Hamanishi C: Cyclic tensile stretch loaded on bovine chondrocytes causes depolymerization of hyaluronan: involvement of reactive oxygen species. Arthritis Rheum 48: 3151-3158, 2003.

32. Trujillo E, Alvarez de la Rosa D, Mobasheri A, Avila J, Gonzalez T and Martin-Vasallo P: Sodium transport systems in human chondrocytes. Morphological and functional expression of the $\mathrm{Na}^{+}, \mathrm{K}^{+}$-ATPase alpha and beta subunit isoforms in healthy and arthritic chondrocytes. Histol Histopathol 14: 1011-1022, 1999.

33. Mobasheri A: Regulation of $\mathrm{Na}^{+}, \mathrm{K}^{+}$-ATPase density by the extracellular ionic and osmotic environment in bovine articular chondrocytes. Physiol Res 48: 509-512, 1999. 